University of Nebraska - Lincoln

DigitalCommons@University of Nebraska - Lincoln

$4-11-1994$

\title{
Parallels between high doubly excited state spectra in $\mathrm{H}^{-}$and $\mathrm{Li}^{-}$ photodetachment
}

\author{
Cheng Pan \\ University of Nebraska - Lincoln \\ Anthony F. Starace \\ University of Nebraska-Lincoln, astarace1@unl.edu \\ Chris H. Greene \\ University of Colorado, Boulder, chgreene@purdue.edu
}

Follow this and additional works at: https://digitalcommons.unl.edu/physicsstarace

Part of the Physics Commons

Pan, Cheng; Starace, Anthony F.; and Greene, Chris H., "Parallels between high doubly excited state spectra in $\mathrm{H}^{-}$and $\mathrm{Li}^{-}$photodetachment" (1994). Anthony F. Starace Publications. 158.

https://digitalcommons.unl.edu/physicsstarace/158

This Article is brought to you for free and open access by the Research Papers in Physics and Astronomy at DigitalCommons@University of Nebraska - Lincoln. It has been accepted for inclusion in Anthony F. Starace Publications by an authorized administrator of DigitalCommons@University of Nebraska - Lincoln. 
Published in Journal of Physics B: Atomic, Molecular and Optical Physics 27:8 (April 28, 1994), pp. L137-L142. Copyright (C) 1994 Institute of Physics Publishing. Used by permission. http://www.iop.org/EJ/journal/JPhysB

Submitted February 23, 1994

LETTER TO THE EDITOR

\title{
Parallels between high doubly excited state spectra in $\mathrm{H}^{-}$and $\mathrm{Li}^{-}$photodetachment
}

\author{
Cheng Pan, ${ }^{1}$ Anthony F. Starace, ${ }^{1}$ and Chris H. Greene ${ }^{2}$ \\ ${ }^{1}$ Department of Physics and Astronomy, University of Nebraska-Lincoln, \\ Lincoln, NE 68588-0111, USA \\ ${ }^{2}$ Department of Physics and Joint Institute for Laboratory Astrophysics, Uni- \\ versity of Colorado, Boulder, CO 80309-0440, USA
}

\begin{abstract}
Eigenchannel $R$-matrix calculations for photodetachment of $\mathrm{H}^{-}$up to the $\mathrm{H}(n=6)$ threshold provide complete theoretical confirmation of observed high-lying, doubly excited state spectra. Long-range multipole interactions beyond the reaction volume are found to play an important role. Similar calculations for $\mathrm{Li}^{-}$photodetachment up to the $\mathrm{Li}(n=6)$ threshold predict analogous resonance structures. These results indicate that recent advances in understanding two-electron correlations in $\mathrm{H}^{-}$and He have application to many electron systems.
\end{abstract}

Experimental measurements of doubly-excited state atomic spectra have long served as stimuli for novel theoretical descriptions of correlated electronic states. Indeed, the first measurements of He doubly-excited state spectra below the $\mathrm{He}^{+}(n=2)$ threshold (Madden and Codling 1963) led theorists to abandon the independent-particle model in order to properly describe the observed experimental intensities (Cooper et al. 1963, Macek 1968). The first theoretical treatment (Cooper et al. 1963) introduced the so-called "+" and "-“ doubly-excited state descriptions, which represented linear combinations of independent particle states having either enhanced (" + ") or diminished ("-") probability amplitude in the vicinity of the atomic ground state and which thereby corresponded respectively to the experimentally observed (Madden and Codling 1963) strong and weak series of doubly- excited states. Macek's adiabatic hyperspherical coordinate treatment generalized this theoretical description and provided a framework for understanding all experimental details (Macek 1968). Recent experimental measurements of doubly-excited state spectra for $\mathrm{H}^{-}$(Harris et al. 1990) and for He (Domke et al. 1991) in the vicinity of much higher detachment or ionization thresholds (i.e., below the $\mathrm{H}(n)$ or $\mathrm{He}^{+}(n)$ thresholds, where $n>2$ ) have been interpreted by theorists as reflecting propensity rules for populating particular channels of "+"-type doubly-excited states (Sadeghpour and Greene 1990, Rost and Briggs 1990, Rost et al. 1991, Sadeghpour 1991, Sadeghpour et al. 1992, Sadeghpour and Cavagnero 1993). Even weak experimental features have been successfully described theoretically (Tang et al. 1992). So far, however, all of these experimental and theoretical advances have focused on the $\mathrm{He}$ and $\mathrm{H}^{-}$two-electron systems, as these represent the prototypes for the study of correlated electronic states. Furthermore, experiment has generally led theory in these developments.

In this letter, we report that such theoretical advances in understanding of doubly-excited state spectra of two-electron systems have application to understanding 
doubly-excited state spectra of a four-electron system, $\mathrm{Li}^{-}$. The eigenchannel $R$-matrix method (Fano and Lee 1973, O'Mahony and Greene 1985, Greene and Kim 1987, Greene 1988) has been used to calculate photodetachment cross sections for both $\mathrm{H}^{-}$ and $\mathrm{Li}^{-}$up to the $n=6$ thresholds (i.e., $\mathrm{H}(n=6)$ and $\mathrm{Li}(n=6)$ ). In order to obtain converged results successfully, the close-coupling equations without exchange were solved outside the $R$-matrix box, thereby treating long-range multipole effects. Evidence for the accuracy of our method is given by our results for $\mathrm{H}^{-}$, which provide the first $a b$ initio theoretical confirmation of experimental results below the $\mathrm{H}(n=5)$ and $\mathrm{H}(n=6)$ thresholds (Harris et al. 1990). Comparisons of the calculated doubly-excited state spectra and selected density plots reveal that propensity rules postulated for doubly-excited state spectra in two-electron systems (Sadeghpour and Greene 1990, Rost and Briggs 1990, Rost et al. 1991, Sadeghpour 1991, Sadeghpour et al. 1992, Tang et al. 1992, Sadeghpour and Cavagnero 1993) are applicable to the description of the $\mathrm{Li}^{-}$doubly-excited state spectrum. Doubly-excited states which violate these propensity rules are shown to cause only very subtle features in the theoretical cross sections for $\mathrm{H}^{-}$and $\mathrm{Li}^{-}$. Experimental measurements of $\mathrm{Li}^{-}$photodetachment in the vicinity of such high $\operatorname{Li}(n)$ thresholds have yet to be performed.

The eigenchannel $R$-matrix method (Fano and Lee 1973, O'Mahony and Greene 1985, Greene and Kim 1987, Greene 1988) aims to determine variationally a set of normal logarithmic derivatives of a system's wavefunction that are constant across a reaction surface $S$ enclosing a reaction volume $V$. For treatments of two-electron excitations, the reaction volume $V$ is that part of six-dimensional configuration space for which both electrons lie within a sphere of radius $r_{0}$. The reaction surface $S$ is the set of points for which $\max \left(r_{1}, r_{2}\right)=r_{0}$, where $r_{1}$ and $r_{2}$ are the electron distances from the nucleus. In practice, for each range of excitation energy, $r_{0}$ is chosen to be sufficiently large that the probability of both electrons being outside $r_{0}$ is negligible. The complicated many-electron interactions within $V$ are treated by bound-state, configuration interaction techniques using independent electron functions and LS-coupling. Normally $r_{0}$ is also chosen large enough so that long range interaction effects may be neglected. For $\mathrm{H}^{-}$, the degeneracy of final state $\mathrm{H}$ atom levels does not permit this. In the work of Sadeghpour et al. (1992) and Sadeghpour and Cavagnero (1993), such effects were treated analytically within the dipole representation (Seaton 1961, Gailitis and Damburg 1963). In this work, all long-range multipole interactions were treated numerically by close-coupling procedures for both $\mathrm{H}^{-}$and $\mathrm{Li}^{-}$(Pan et al. 1994). This permitted much smaller values of $r_{0}$ to be used than would otherwise be the case: 80 au for spectra below the $n=5$ threshold and 100 au for spectra below the $n=6$ threshold. These box sizes nevertheless are large enough to ensure that the $\mathrm{H}(n=5)$ and $\mathrm{H}(n$ $=6$ ) energy manifolds are degenerate to within a fraction of $1 \mathrm{meV}$.

Our results for photodetachment of $\mathrm{H}^{-}$with excitation of $\mathrm{H}(n=4)$ and $\mathrm{H}(n=5)$ are shown in Figure 1(a) and (b) respectively, together with the experimental results of Harris et al. (1990). The theoretical results have been convoluted with a Gaussian energy function to take into account the experimental energy resolution of $8.3 \mathrm{meV}$. Both the length and velocity form theoretical results are in excellent agreement with the experimental results up to the energy of the second window resonance in each case (i.e., up to $\approx 13.76 \mathrm{eV}$ in (a) and $\approx 13.93 \mathrm{eV}$ in (b)). The discrepancies beginning at these second window resonances, however, merely indicate that as one approaches the $\mathrm{H}(n=$ $5)$ and $\mathrm{H}(n=6)$ thresholds, one requires a larger $R$-matrix box to treat the increasingly broad radial extensions of the higher energy resonances. The complete $\mathrm{H}(n=4)$ spectrum below the $\mathrm{H}(n=5)$ threshold - cf. Figure 1(a) - and that for $\mathrm{H}(n=5)$ below the $\mathrm{H}(n=6)$ threshold - cf. Figure $1(b)$ - have been calculated using $R$-matrix radii of 80 $\mathrm{au}$ and $100 \mathrm{au}$ respectively. In order to demonstrate that we can improve the results near and above the second window resonances in each case, we show more extensive calculations in the insets employing $R$-matrix radii of 100 au and 120 au respectively. 

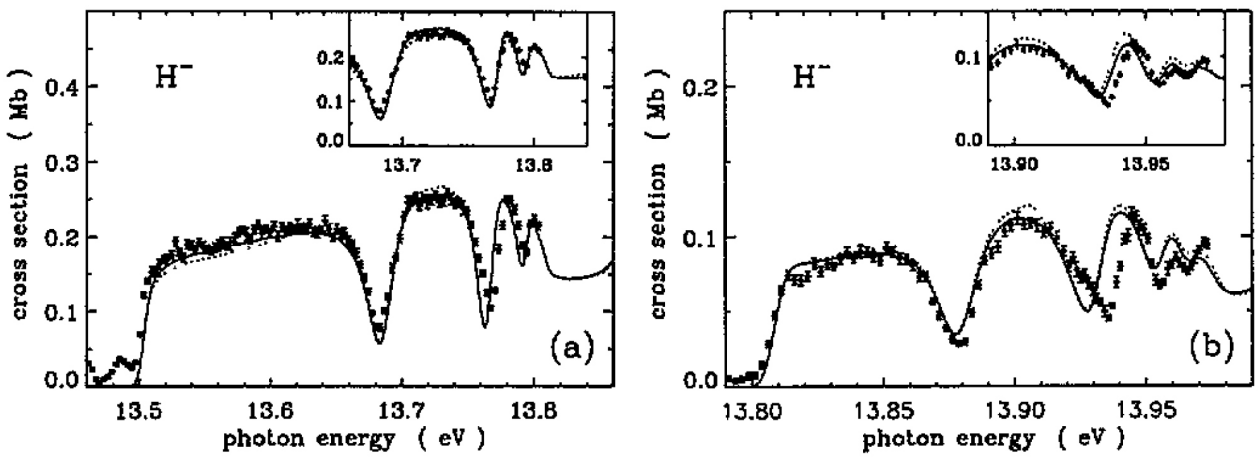

Figure 1. Photodetachment cross sections for the processes $\mathrm{H}^{-}+\gamma \rightarrow \mathrm{H}(n)+\mathrm{e}^{-}$plotted against the photon energy, where (a) $n=4$ and (b) $n=5$. Theoretical curves: Present results using the velocity form (full curve) and length form (dotted curve) of the dipole operator. Note that in this figure the theoretical results have been convoluted with the experimental resolution of $8.3 \mathrm{meV}$. Inset figures show results of more extensive theoretical calculations: see text for details. Experimental points: Harris et al. (1990).

In these insets, one sees that the theoretical $\mathrm{H}(n=4)$ partial cross sections are now in excellent agreement with experiment up to the third window resonance near 13.78 $\mathrm{eV}$; also, the theoretical $\mathrm{H}(n=5)$ results now agree with experiment over the low-energy half of the second window resonance.

Turning now to the comparison of $\mathrm{H}^{-}$and $\mathrm{Li}^{-}$photodetachment, Figures 2 and 3 compare the partial cross sections for exciting respectively the $n=4$ and $n=5$ states of the neutral atom. To facilitate comparison, the spectra are plotted against energy relative to the double ionization threshold. One sees that, on a coarse energy scale, the two spectra are very similar, particularly as one approaches the double ionization threshold. The prominent series of window resonances (whose first members are labeled by $a$ and $a^{\prime}$ ) are strong features of both spectra. The weak features (whose first members are labeled $b, b^{\prime}$, and $c$ ) are, however, different in the two spectra. These differences probably stem from the exact degeneracy of the $\mathrm{H}$ atom energy levels as compared to the lack of degeneracy in the Li atom. Thus, whereas the series of resonances
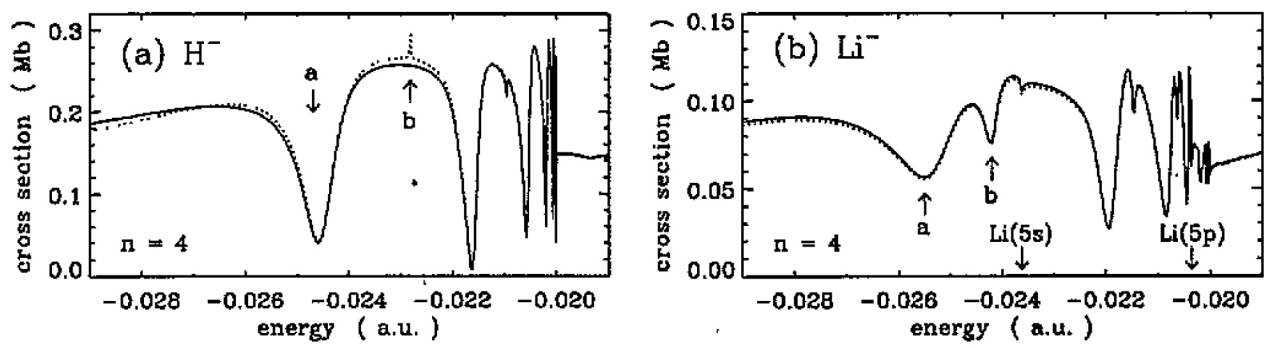

Figure 2. (a) Photodetachment cross sections for the process $\mathrm{H}^{-}+\gamma \rightarrow \mathrm{H}(n=4)+\mathrm{e}^{-}$. (b)Photodetachment cross sections for the process $\mathrm{Li}^{-}+\gamma \rightarrow \operatorname{Li}(n=4)+\mathrm{e}^{-}$. The abscissae show the final state energy relative to the double ionization threshold. Full curves $=$ present dipole velocity results; broken curves $=$ present dipole length results. Labels $a$ and $b$ denote the doubly-excited resonances ${ }_{5}\{0\}_{5}^{+}$and ${ }_{5}\{0\}_{6}^{-}$respectively. The cusplike structure in (b) at the energy -0.02364 is located at the $\mathrm{Li}(5 \mathrm{~s})$ threshold. 

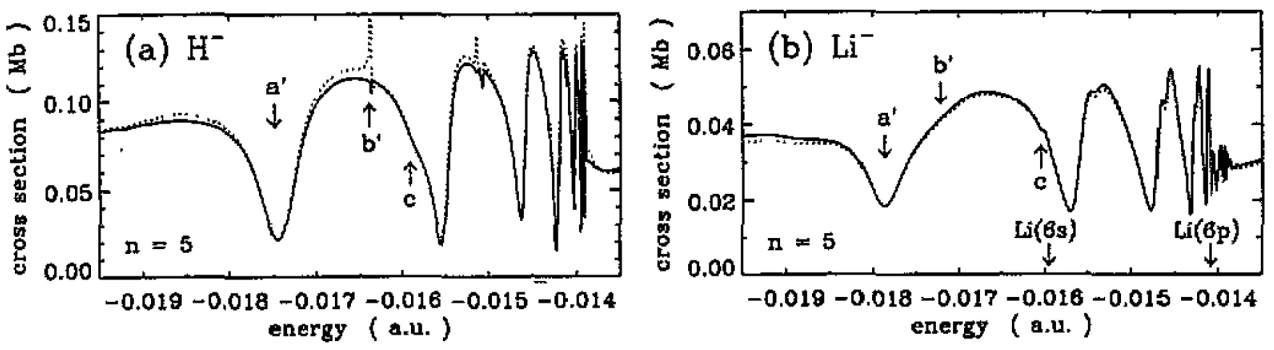

Figure 3. (a) Photodetachment cross sections for the process $\mathrm{H}^{-}+\gamma \rightarrow \mathrm{H}(n=5)+\mathrm{e}^{-}(\mathbf{b})$ Photodetachment cross sections for the process $\mathrm{Li}^{-}+\gamma \rightarrow \operatorname{Li}(n=5)+\mathrm{e}^{-}$. The abscissae show the final state energy relative to the double ionization threshold. Full curves = present dipole velocity results; broken curves $=$ present dipole length results. Labels $a^{\prime}$, $b^{\prime}$, and $c$ denote the doubly-excited resonances ${ }_{6}\{0\}_{6}^{+},{ }_{6}\{0\}_{7}^{-}$, and ${ }_{6}\{1\}_{6}^{+}$, respectively.

whose first two members are labeled $b$ and $b^{\prime}$ are broad features of the $\mathrm{Li}^{-}$spectrum (even though very subtle in the case of $b^{\prime}$ ), they are extremely narrow features of the $\mathrm{H}^{-}$spectrum. (Note that $b$ does not even show up in the dipole velocity results in Figure $2(\mathrm{a})$.)

In order to make connection with predicted propensity rules and to identify the features in the calculated cross sections, special $R$-matrix calculations were carried out with an interaction volume of radius $r_{0}=120 \mathrm{au}$. All basis functions were set to zero on the boundary of $V$. Thus only the discrete structures were calculated, in order to see which ones appeared at energies corresponding to the features seen in the cross sections shown in Figures 2 and 3 . In the case of the $n=5$ spectra, doubly excited state resonances were found at the locations indicated by the labels $a$ and $b$ in Figure 2. The $a$ resonance has a peak probability amplitude at a hyperspherical radius $R \approx 60$ au. The $b$ resonance has a peak probability amplitude at a hyperspherical radius $R \approx 80$ $\mathrm{au}$. The wavefunctions for these resonances were then used to make density plots in hyperspherical coordinates, spheroidal coordinates, and $\left(r_{1}, r_{2}\right)$ coordinates, which are compared elsewhere (Pan et al. 1994). For every method of plotting the densities, the structures of the corresponding resonances for $\mathrm{H}^{-}$and $\mathrm{Li}^{-}$were found to be identical.

The hyperspherical coordinate plots in $\alpha\left(=\tan ^{-1}\left(r_{2} / r_{1}\right)\right)$ and $\theta_{12}$ are shown for the resonances labeled $a$ and $b$ in Figures 4 and 5 respectively. These density plots can be described most simply in the notation ${ }_{N}\{v\}_{n}^{A}$ introduced in the work of Sadeghpour (1991) and Sadeghpour et al. (1992). In this notation, $N$ denotes the principal quantum
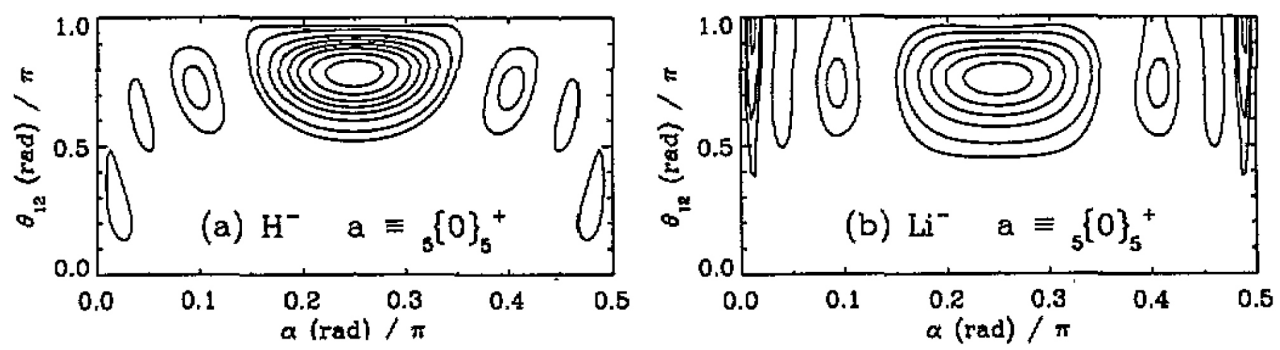

Figure 4. Doubly-excited state $a={ }_{5}\{0\}_{5}^{+} 5(0)$ : wavefunction density plotted against $\alpha$ and $\theta_{12}$. (a) $\mathrm{H}^{-}$, (b) $\mathrm{Li}^{-}$. 

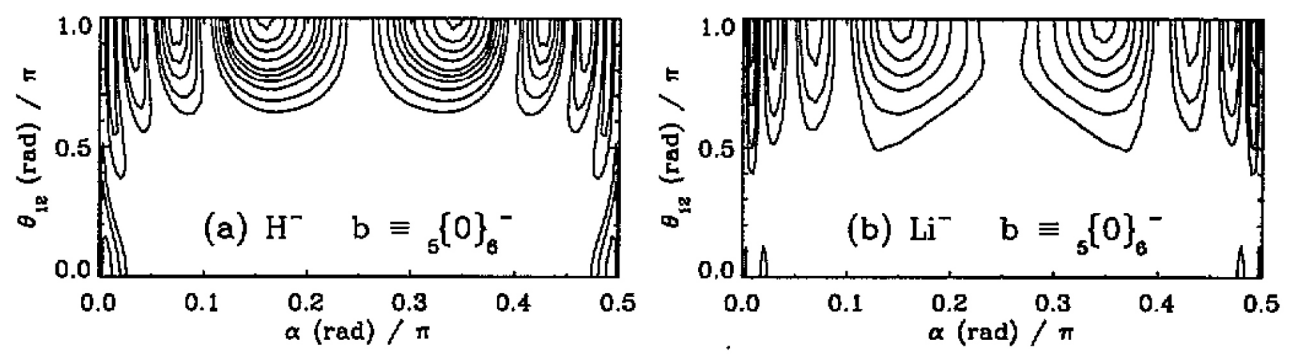

Figure 5. Doubly-excited state $b={ }_{5}\{0\}_{6}^{-}$wavefunction density plotted against $\alpha$ and $\theta_{12} \cdot(\mathbf{a}) \mathrm{H}^{-},(\mathbf{b}) \mathrm{Li}^{-}$.

number of the threshold to which the resonance series is converging. $v$ is a vibrational quantum number introduced by Watanabe and Lin (1986) and defined by $1 / 2(N-1-K$ - T), where $K$ and $T$ are quantum numbers introduced by Herrick $(1975,1983)$ and Lin $(1984,1986) ; v$ indicates the number of nodes in $\theta_{12}$. A takes the values + and - , which denote the presence of a two-electron wavefunction antinode or node respectively for $r_{1}=r_{2}$ or, equivalently, $\alpha=\pi / 4$ (Cooper et al. 1963, Lin 1984, 1986). Finally, $n$ denotes the principal quantum number of the second electron of the doubly excited state. Examining the density plots, one sees clearly that $a$ is a ${ }_{5}\{0\}_{5}^{+}$resonance, whereas $b$ is a ${ }_{5}\{0\}_{6}^{-}$resonance.

The $a^{\prime}$ and $b^{\prime}$ resonances indicated in Figure 3 have been similarly calculated. Examination of the density plots (not shown) indicates that these resonances are ${ }_{6}\{0\}_{6}^{+}$ and ${ }_{6}\{0\}_{7}^{-}$respectively. They are simply the next members of their series, having one more node in alpha on either side of $\alpha=1 / 4 \pi$ than do the lower members $a$ and $b$ whose densities are shown in figures 4 and 5. Significantly, the $b^{\prime}$ resonance in $\mathrm{Li}^{-}$has less amplitude on the $r_{1}=r_{2}$ ridge than does the $b$ resonance shown in Figure 5(b).This indicates why the $b^{\prime}$ feature in Figure $3(b)$ is so much less prominent than is $b$ in Figure 2(b).

The resonance $c$ indicated in Figure 3 was found to have a maximum probability amplitude at a hyperspherical radius $R \approx 90$ au. Its density plot, shown in Figure 6 , indicates that it is $\mathrm{a}_{6}\{1\}_{6}^{+}$resonance. Once again, the density plots show the resonances in $\mathrm{H}^{-}$and $\mathrm{Li}^{-}$to be very similar, even though their effect on the cross sections in Figure 3 is rather different.

In summary, eigenchannel $R$-matrix calculations including effects of long-range multipole interactions outside the $R$-matrix box have been carried out for photodetachment of $\mathrm{H}^{-}$and $\mathrm{Li}^{-}$up to the $n=6$ threshold. Results for $\mathrm{H}^{-}$give the first com-
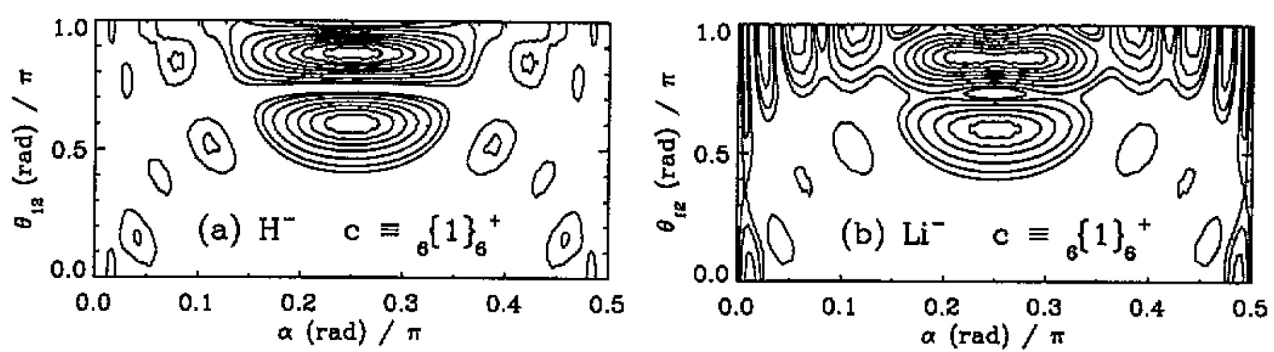

Figure 6. Doubly-excited state $c={ }_{6}\{1\}_{6}^{+}$wavefunction density plotted against $\alpha$ and $\theta_{12} \cdot(\mathbf{a}) \mathrm{H}^{-},(\mathbf{b}) \mathrm{Li}^{-}$. 
plete theoretical confirmation of experimental measurements (Harris et al. 1990) below the $n=5$ and $n=6$ thresholds. Partial cross sections for $\mathrm{Li}^{-}$are found to be remarkably similar to those for $\mathrm{H}^{-}$, despite the absence of atomic energy level degeneracy in the case of Li. Part of the reason for this similarity is undoubtedly the fact that only swave quantum defects are large for Li. Density plots for two electron resonances appearing in each spectrum, confirm the similarity of the two spectra, indicating that advances in understanding doubly-excited states in two-electron systems have application to doubly-excited state spectra in many-electron systems. A more complete description of the present results for $\mathrm{Li}^{-}$photodetachment. including presentation of the full spectrum from the $n=2$ to the $n=6$ thresholds, will be given elsewhere (Pan et al. 1994).

\section{Acknowledgments}

This work was supported in part by the US Department of Energy, Division of Chemical Sciences, Office of Basic Energy Sciences under grant no. DE-FG02-88ER139.55 at the University of Nebraska and grant no DE-FG02-90ER14145 at the University of Colorado.

\section{References}

Cooper, J. W., Fano, U., and Pratts, F. 1963 Phys. Rev. Lett. 10: 518-21.

Domke, M., Xue, C., Puschmann, A., Mandel, T., Hudson, E., Shirley, D. A., Kaindl, G., Greene, C. H., Sadeghpour, H. R., and Petersen, H. 1991 Phys. Rev. Lett. 66: 1306-9.

Fano, U., and Lee, C. M. 1973 Phys. Rev. Lett. 31: 1573-6.

Gailitis, M., and Damburg, R. 1963 Proc. Phys. Soc. 82: 192-200.

Greene, C. H. 1988 Fundamental Processes of Atomic Dynamics, ed. J. S. Briggs, H. Meinpoppen, and H. O. Lutz (New York, Plenum) pp. 105-27.

Greene, C. H., and Kim L. 1987 Phys. Rev. A 36: 2706-17.

Harris, P. G., Bryant, H. C., Mohagheghi, A. H., Reeder, R. A., Sharifian, H., Tang, C. Y., Tooloonchi, H., Donahue, J. B., Quick, C. R., Rislove, D. C., Smith, W. W., and Stewart, J. E. 1990 Phys. Rev. Lett. 65: 309-12.

Herrick, D. R. 1975 Phys. Rev. A 12: 412-24. 1983 Adv. Chem. Phys. 52: 1-115.

Lin, C. D. 1984 Phys. Rev. A 29: 1019-33. 1986 Adv. At. Mol. Phys. 22: 77-142

Macek, J. H. 1968 J. Phys. B: At. Mol. Phys. 1: 831-43.

Madden, R. P., and Codling, K. 1963 Phys Rev. Lett. 10: 516-18.

O'Mahony, P. F., and Greene, C. H. 1985 Phys. Rev. A 31: 250-59.

Pan, C., Starace, A. F., and Greene, C. H. 1984 to be published.

Rost, J. M., and Briggs, J. S. 1990 J. Phys. B: At. Mol. Opt. Phys. 23: L339-46.

Rost, J. M., Briggs, J. S., and Feagin, J. M. 1991 Phys. Rev. Lett. 66: 1642.

Sadeghpour, H. R. 1991 Phys. Rev. A 43: 5821-31.

Sadeghpour, H. R., and Cavagnero, M. 1993 J. Phys. B: At. Mol. Opt. Phys. 26: L271-74.

Sadeghpour, H. R., and Greene, C. H. 1990 Phys. Rev. Lett. 65: 313-16.

Sadeghpour, H. R., Greene, C. H., and Cavagnero, M. 1992 Phys. Rev. A 45: 1587-95.

Seaton, M. J. 1961 Proc. Phys. Soc. 77: 174-83.

Tang, J. Z., Watanabe, S., Matsuzawa, M., and Lin, C. D. 1992 Phys. Rev. Lett. 69: 1633-35.

Watanabe, S., and Lin, C. D. 1986 Phys. Rev. A 34: 823-37. 